\title{
Relação entre Distribuição dos Rendimentos do Trabalho e Industrialização: Evidências para os Municípios Brasileiros
}

\author{
Patrick Leite Santos ${ }^{1}$ \\ Carlos César Santejo Saiani ${ }^{2}$ \\ Bruno Benzaquen Perosa ${ }^{3}$
}

\begin{abstract}
Resumo
O presente estudo investigou, empiricamente, a relação entre industrialização e distribuição dos rendimentos do trabalho nos municípios brasileiros. Com base em debates históricos sobre distribuição de renda da academia brasileira e nas hipóteses da Curva de Kuznets e, especialmente, a que se refere à economia dual, o estudo encontrou uma relação não linear entre a desigualdade de rendimentos do trabalho e a industrialização, medida pelas participações industriais no produto e no emprego. A partir de dados municipais referentes a 2000 e 2010, foram realizadas regressões paramétricas em painel e não paramétricas. As evidências, relativamente robustas, sugerem que a curva derivada da relação entre a desigualdade de renda e a industrialização tem o formato próximo a um "U-invertido". Ou seja, a distribuição dos rendimentos do trabalho piora com a industrialização até certo de nível de participação industrial (no produto e no emprego); mas, atingido certo nível, a distribuição passa a melhorar.
\end{abstract}

Palavras-chave: Industrialização. Distribuição de Renda. Curva de Kuznets. Dados em Painel.

\begin{abstract}
This paper presented an empirical investigation of the relation between industrialization and labor income on Brazilian municipalities. Based on the Brazilian debates on income distribution and Kuznets curve, specially the dual economy hypotheses, this research found a nonlinear relation between labor income inequality and industrialization, here considered as the share of industrial sector on product and jobs. Considering municipal data from 2000 to 2010, parametric and nonparametric regressions offered relatively robust evidence suggesting that the curve relating inequality and industrialization has a "U-inverted" shape. In conclusion, labor income distribution is aggravated (reduced) when the industrial sector increases its share (on both the product and jobs), but, after a certain level, the distribution starts to get better.
\end{abstract}

Keywords: Industrialization. Income distribution. Curve of Kuznets. Panel Data.

Classificação JEL: O14. O15. R11.

Área ABEIN: Área 1 - Indústria e competitividade;

1.4 Padrões de especialização produtiva e desenvolvimento.

\footnotetext{
${ }^{1}$ Instituto de Economia e Relações Internacionais da Universidade Federal de Uberlândia - IERI/UFU.

${ }^{2}$ Instituto de Economia e Relações Internacionais da Universidade Federal de Uberlândia - IERI/UFU.

${ }^{3}$ Instituto de Economia e Relações Internacionais da Universidade Federal de Uberlândia - IERI/UFU.
} 


\section{Introdução}

Distribuição de renda e industrialização são importantes debates econômicos nos cenários brasileiro e internacional. No contexto atual brasileiro, tais debates ganham destaque em função da concentração da renda ter sofrido uma relativa redução em anos recentes comparativamente a vários anos de expansão contínua. Porém, o país ainda figura entre os piores no que se refere a tal aspecto. Além disso, argumenta-se que o país vem passando por uma desindustrialização.

No cenário acadêmico, as duas temáticas são relacionadas por diferentes abordagens. No Brasil, destaca-se o debate que ficou conhecido como "controvérsia de 70", no qual diferentes vertentes tentaram explicar o aparente caráter concentrador de renda do crescimento econômico impulsionado pela industrialização do período do "Milagre Econômico" no governo militar. Em âmbito mundial, as interfaces entre crescimento econômico, industrialização e desigualdade de renda são discutidas por diferentes teorias, podendo-se destacar a da Curva de Kuznets (CK).

A hipótese da CK pressupõe uma relação no formato próximo a um "U-invertido" entre a desigualdade de renda e o crescimento econômico. Ou seja, em níveis pequenos de renda per capita, a desigualdade de renda aumentaria em função do crescimento; porém, atingido certo nível de renda (turning point), a desigualdade passaria a cair. Tal hipótese embasa, inclusive, argumentos da "controvérsia de 70". Diversos trabalhos contestam a CK, defendendo que a desigualdade de renda teria dinâmica cíclica associada ao crescimento econômico. Dentre estes trabalhos, deve-se destacar aqueles que preconizam uma relação desigualdade-crescimento em formato de um "N"; ou seja, a desigualdade voltaria a aumentar em maiores níveis de renda.

A relação desigualdade-crescimento nos formatos da CK ou do "N" são justificadas por vários aspectos, dentre os quais, argumentos associados à abordagem da economia dual. Para estes, a desigualdade de renda seria influenciada pelo perfil setorial da economia. O aumento e a posterior queda da desigualdade de renda em função do crescimento econômico decorreriam da transição de uma economia baseada em atividades de agropecuária para uma economia com predominância industrial (industrialização). A migração de trabalhadores e os diferenciais de produtividade do trabalho e de rendimentos entre os setores gerariam, no início, aumento da desigualdade; mas, com a consolidação da indústria, a desigualdade diminuiria. Em um segundo momento, a transição de uma economia industrial para uma com maior relevância dos serviços - o que, para a literatura, pode sinalizar uma desindustrialização "natural" - aumentaria a desigualdade devido à migração de trabalhadores e diferenciais de produtividade e rendimentos.

Com base nestes debates, este estudo investiga, empiricamente, como a industrialização afeta a desigualdade dos rendimentos do trabalho nos municípios brasileiros. Especificamente, é averiguado se as relações entre a desigualdade de rendimentos e as participações industriais no produto e no emprego não são lineares, apresentando formato próximo a um "U-invertido". Ou seja, se a desigualdade de renda aumenta em função da industrialização em níveis baixos de participações industriais e passa a diminuir quando são atingidas certas participações (turning points). Tal hipótese é embasada pelas supracitadas discussões da abordagem da economia dual. Além disso, as hipóteses da CK e do "N" também são testadas para os municípios brasileiros.

É importante ressalvar que, segundo a literatura, os impactos da industrialização sobre a desigualdade de renda de uma localidade decorrem de efeitos composição e concentração. $\mathrm{O}$ primeiro é a alteração da desigualdade provocada por alterações da participação industrial na economia. O segundo é a variação da desigualdade por mudanças da concentração de renda na indústria. Evidências de outros trabalhos, com análises estáticas, sinalizam que a indústria, em comparação aos outros grandes setores econômicos, concentra menos renda no Brasil. Assim, 
se ocorresse apenas um efeito composição, o aumento da participação industrial deveria sempre resultar em menor desigualdade de rendimentos do trabalho nos municípios brasileiros. Porém, se for constatada a relação não linear, pode-se inferir que a industrialização impacta sobre a desigualdade dos rendimentos do trabalho por combinações dinâmicas dos efeitos composição e concentração, o que estaria de acordo com os argumentos da abordagem da economia dual.

Para testar as hipóteses, são realizadas estimações paramétricas em painel (efeitos fixos, efeitos aleatórios e Tobit) e não paramétricas (Kernel-Weighted Local Polynomial Regression), para todos os municípios brasileiros com dados disponibilizados referentes aos anos de 2000 e 2010 - últimos em que foram realizados Censos Demográficos no Brasil. Os dados são oriundos do Instituto Brasileiro de Geografia e Estatística (IBGE) e do Programa das Nações Unidas para o Desenvolvimento (PNUD). Como medida de desigualdade de renda, é usado o índice Theil-L dos rendimentos do trabalho - assim, o estudo insere-se no debate sobre a distribuição pessoal e setorial da renda. Seguindo a literatura, são adotadas duas medidas de (des)industrialização: i) participação do valor adicionado (VA) pela indústria no Produto Interno Bruto (PIB); e ii) participação industrial no emprego. Já como medida de renda per capita, é utilizado o PIB per capita. Vale destacar que são adotadas algumas estratégias para testar a robustez dos resultados.

Antecipadamente, deve-se apontar que as evidências encontradas não refutam a hipótese derivada da economia dual de que a relação desigualdade-industrialização tem, nos municípios brasileiros, um formato próximo a um "U-invertido", tanto para a participação industrial no produto como no emprego. Assim, a desigualdade dos rendimentos do trabalho aumenta com a industrialização até certas participações da indústria, a partir das quais, cai. A não linearidade das relações obtidas sugere que o impacto da indústria na desigualdade de rendimentos não advém apenas de um efeito composição, mas da combinação deste com um efeito concentração.

Outras evidências que devem ser destacadas: i) os turning points, tanto para a participação industrial no produto como no emprego, são superiores às respectivas participações médias; ii) os turning points da participação industrial no emprego são menores do que os da participação no produto; iii) a relação desigualdade-crescimento apresenta um formato próximo a um "N"4.

Estes resultados contribuem para a literatura. Por um lado, preenchendo uma lacuna na literatura empírica, ao menos para os municípios brasileiros, pois poucos trabalhos averiguaram explicitamente a relação desigualdade-industrialização ao invés da desigualdadecrescimento nos formatos da CK ou "N", que utilizam a economia dual apenas como possível explicação ${ }^{5}$. Por outro lado, garante evidências adicionais à literatura sobre consequências favoráveis da industrialização, que enfatiza impactos no crescimento econômico: i) efeitos de encadeamento nas cadeias produtivas; ii) produtividade industrial crescente em função da produção do setor; iii) indução e difusão de progresso tecnológico; e iv) elasticidades-renda das importações de bens industriais superiores às de commodities e primários, aliviando o balanço de pagamentos ${ }^{6}$.

Além desta introdução e das considerações finais, o presente estudo está estruturado em 3 seções. Na primeira, é realizada uma breve revisão da literatura sobre os possíveis efeitos da industrialização sobre a desigualdade de renda, destacando debates da academia brasileira e

\footnotetext{
${ }^{4}$ Evidências coerentes às de Figueiredo et al. (2011), também para os municípios brasileiros com outras estratégias.

${ }^{5} \mathrm{Na}$ verdade, foi encontrado apenas o trabalho de Bêrni et al. (2002) que testou, somente para municípios do estado do Rio Grande do Sul, a hipótese da CK substituindo a medida de renda per capita pelo valor adicionado da indústria, mas não sua participação no produto e nem a participação industrial no emprego.

${ }^{6}$ Para mais detalhes, conferir, entre outros: Kaldor (1966) e Oreiro e Feijó (2010).
} 
em âmbito mundial que fundamentam as hipóteses da abordagem da economia dual. Na segunda, as estratégias empíricas e os dados são apresentados. Na terceira, os resultados são analisados.

\section{Referencial histórico, teórico e empírico}

\subsection{Debates sobre a industrialização e a distribuição de renda no Brasil}

No Brasil, a industrialização passou a ser mais debatida a partir dos anos 1930, no governo do Presidente Getúlio Vargas, caracterizado pelo avanço da indústria por indução estatal. Tal indução encontra na literatura várias justificativas, desde trabalhos que a entendiam como uma forma de proteção e diversificação do capital cafeeiro, embasados no livro de Celso Furtado, "Formação Econômica do Brasil" (FURTADO, 1967), até trabalhos que defendiam mudança na estrutura produtiva provocada por ações estatais para dinamizar o mercado interno a partir da indústria. A defesa do desenvolvimento pautado na industrialização era fundamentada por alguns exemplos favoráveis, como os Estados Unidos (EUA) e a Inglaterra (FONSECA, 2003).

Na década de 1950, a tese predominante passou a ser a "cepalina", que identificava a "deterioração dos termos de troca" como nociva para países com pauta exportadora dependente de commodities agrícolas, o que geraria déficits nas transações internacionais. Em resposta a tal situação, a escola cepalina recomendava para os países Latinos Americanos a implementação de políticas de industrialização por substituição de importações (PREBISCH, 1962).

Políticas deste tipo foram adotadas no Brasil de 1950 até a década de 1980, influenciadas e direcionadas por um "Estado Desenvolvimentista" com as funções de induzir e sinalizar investimentos e, até mesmo, de investir e produzir diretamente visando à industrialização. O auge destas políticas ocorreu de 1968 a 1973, durante o regime militar, no período conhecido como "Milagre Econômico" devido às significativas expansões da industrialização e do produto agregado (TAVARES, 1975; BAER, 1993; CARNEIRO, 1990; LAGO, 1990; SILVA, 2003).

Na década de 1980, o modelo de substituição de importações esgotou-se e os principais debates sobre o tema passaram a ser: i) possível desindustrialização, causas e consequências; e ii) desconcentração espacial da indústria. No primeiro, havia duas posições. Uma defendia a hipótese de desindustrialização "induzida" por "doença holandesa", que teria sido intensificada pelas políticas de abertura comercial e financeira. Outra defendia a desindustrialização como um fenômeno "natural" devido à expansão dos serviços. Assim, diferenças da produtividade do trabalho e da elasticidade-renda explicariam a desindustrialização (OREIRO; FEIJÓ, 2010).

Em relação ao segundo debate, Cano (1981) aponta que a industrialização brasileira foi espacialmente desigual até os anos 1970, concentrando-se na região Sudeste, especialmente em São Paulo. A tendência de concentração foi, em parte, revertida pelo "Milagre Econômico" e II Plano Nacional de Desenvolvimento, devido a ações federais. A posteriori, a desconcentração parcial decorreu de "guerra fiscal" nas esferas subnacionais e de decisões das empresas em busca de vantagens comparativas, como custos e mercados (CANO, 1997, SABOIA, 2001).

Voltando a atenção para a distribuição de renda no Brasil, pode-se dizer que, em certo grau e dados momentos, ela foi atrelada à dinâmica da indústria. Por exemplo, a concentração espacial da indústria também resultou em distribuição desigual da renda no território brasileiro (CANO, 1981). O já mencionado livro de Furtado (1967) é pioneiro no assunto ao advogar que a predominância do setor agrário na fase inicial de desenvolvimento brasileiro 
era regida sob sistema de concentração de propriedades e outras atividades nas mãos de poucos. Contudo, tal debate só ganhou maior destaque no "Milagre Econômico", dado que o crescimento induzido pela industrialização foi acompanhado por pior distribuição de renda (LANGONI, 1973).

Nesse contexto, emergiu o debate conhecido como "controvérsia de 70", no qual algumas visões tentaram explicar a piora da desigualdade de renda. Segundo Gandra (2005), o debate foi composto por três vertentes de interpretações. A primeira baseava-se na "Teoria do Capital Humano". Nessa linha, Langoni (1973) defendeu a elevação da desigualdade como decorrência de um déficit de mão-de-obra qualificada. $\mathrm{O}$ baixo número de profissionais qualificados teria superestimado os salários destes, elevando a diferença em relação aos dos menos qualificados. Um agravante desta situação seria a estrutura produtiva da economia brasileira, com setor rural atrasado comparativamente ao industrial, o que resultava em diferenças salariais entre estes setores. Tal abordagem está alinhada à de economia dual, que é discutida na próxima subseção.

A segunda vertente é representada pelos trabalhos de Fishlow $(1972,1978)$ e Hoffmann e Duarte (1972), para os quais, as principais causas do aumento da concentração de renda foram as políticas do governo militar, que provocaram compressão salarial. É o caso do Plano de Ação Econômica do Governo, que alterou a forma de reajuste do salário mínimo. Este passou a tomar como referência a inflação projetada pelo governo, que, quase sempre, era inferior à efetiva. Como o formato de governo não permitia greves ou pressões para negociar a alteração da forma de reajuste, houve queda do poder de compra do salário mínimo. Assim, o decorrente processo inflacionário, junto com o crescimento econômico e o arrocho salarial redistribuíram a renda em favor de alguns setores urbanos (trabalhadores administrativos e financeiros) e empresários.

Já a terceira vertente teve como referência os trabalhos de Bacha (1978) e Bacha e Taylor (1980), que utilizaram argumentos da sociologia e da organização da estrutura hierárquica das empresas. Para formalizarem a interpretação, dividiram os trabalhadores em duas categorias: i) white-collars; e ii) blue-collars. A primeira era composta por trabalhadores de alta qualificação com remunerações atreladas aos resultados da empresa; a segunda, por trabalhadores em geral, com salários definidos pela demanda e oferta no mercado de trabalho. A diferença nas formas de determinação dos salários criava um "leque salarial", que elevava a desigualdade de renda.

Em paralelo ao debate da "controvérsia de 70", outros trabalhos tentaram explicar porque a industrialização contribuiu para a piora da distribuição de renda. Destacam-se Tavares (1975) e Mello (1986), que entendiam que a concentração de renda já estava presente na produção brasileira, mas a industrialização a aprofundou e escancarou. Estes autores, em parceria com outros da "escola de Campinas", defendiam que a concentração piorou devido à forma como se deu a industrialização, que priorizou alguns setores com características concentradoras, como o de bens duráveis. Logo, entendia-se que o crescimento induzido pela industrialização não iria reduzir automaticamente o nível de desigualdade de renda (CARDOSO; POCHMANN, 2000).

Nas décadas seguintes, o debate sobre a industrialização como "causa" da concentração de renda foi deixado um pouco de lado e outras discussões ganharam evidência. Nos anos 1990, destacam-se as visões "estrutural integrada", focada no papel da inflação, e "unilateralista", que retoma, em parte, aspectos de Langoni (1973) - fundamentação na Teoria do Capital Humano e na heterogeneidade educacional (BONELLI; RAMOS, 1993; BARROS et al., 2000; ROCHA et al., 2013). Nos anos 2000, a atenção voltou-se para a redução observada na desigualdade. As interpretações enfatizaram efeitos de políticas de distribuição de renda, valorização do salário mínimo e educacionais (HOFFMANN; NEY, 2008; DEDECCA, 2015; BARROS et al., 2007). 


\subsection{Hipóteses da relação desigualdade-crescimento: a abordagem dual}

Além dos debates internos realizados pela academia brasileira, discutidos anteriormente, existe um debate em âmbito internacional que tenta entender a dinâmica entre industrialização, crescimento econômico e distribuição de renda. Uma das teorias mais discutidas é a "Curva de Kuznets", que, inclusive, embasa um dos argumentos de Langoni (1973) para explicar a concentração de renda no "Milagre Econômico" - atraso do setor rural em relação ao industrial.

Kuznets (1955), com dados referentes aos séculos XIX e XX da Inglaterra, Alemanha e EUA, mostrou evidências que sugerem elevação da desigualdade de renda com o crescimento econômico em níveis menores de renda per capita e, a partir de certo nível, a desigualdade cai. Ou seja, existiria uma relação próxima a um "U-invertido" entre desigualdade e nível de renda. Tal potencial relação ficou conhecida como teoria (ou hipótese) da Curva de Kuznets (CK).

Diversos trabalhos testaram a CK, parte deles levantando algumas possíveis justificativas. Na survey de Deutsch e Silber (2000), as possíveis justificativas são divididas em três grupos, segundo a visão adotada: i) economia dual; ii) crescimento endógeno (ou newgrowth); e iii) teoria da escolha pública. O último e, principalmente, o primeiro são os que aqui merecem destaque por associarem a dinâmica da industrialização como um dos condicionantes da $\mathrm{CK}^{7}$.

Para a abordagem dual, que foi, inclusive, usada como explicação no trabalho de Kuznets (1955), a migração de trabalhadores entre setores da economia, do menos para o mais dinâmico (ou do tradicional para o moderno), causaria, inicialmente, aumento da desigualdade de renda; mas a consolidação do novo setor levaria a queda da desigualdade. Tal dinâmica ocorreria na transição de uma economia com predominância da agropecuária para uma industrial. Assim, seria atrelada à industrialização. No início do processo, a produtividade marginal do trabalho e, consequentemente, os rendimentos na indústria aumentam mais rapidamente, aumentando a desigualdade de renda. Porém, à medida que a industrialização se consolida, com migração de grande parte dos trabalhadores, motivados pelo diferencial de salário, a desigualdade cairia.

Lewis (1954) e Ramos (1991), baseados em modelos de surplus labor, apresentam uma interpretação alternativa pela abordagem dual. A concentração de renda inicial em função do crescimento econômico se dá a uma taxa maior de lucros no setor moderno, que, em relação ao tradicional, está em processo de expansão. Ao longo do tempo, com a economia atingindo o ponto no qual o trabalho passa a ser remunerado segundo a produtividade marginal no setor tradicional, o sistema assume características neoclássicas e a desigualdade tenderia a reduzir.

Os trabalhos relacionados à teoria da escolha pública fundamentam seus argumentos na interação entre mecanismo político e estrutura econômica. Segundo Glaeser (2005), o efeito da industrialização sobre a distribuição de renda seria por meio de políticas redistributivas, que tendem a ser mais adotadas à medida que aumenta a urbanização - associada à expansão da participação da indústria na economia -, o que resulta em maior proximidade entre as pessoas e, assim, maiores capacidades de organização e pressão sobre governantes para o atendimento de suas demandas (controle social). A maior organização também pode gerar

\footnotetext{
7 O principal argumento dos trabalhos do segundo grupo é que, com baixos níveis de renda per capita, imperfeições nos mercados de crédito e distribuições desiguais do capital humano e, consequentemente, da renda são condições para a economia se desenvolver. Ao longo do desenvolvimento, há "transbordamento" de conhecimento para os mais pobres, caindo a desigualdade (AGHION; BOLTON, 1992; GALOR; TSIDDON, 1996).
} 
maior poder de barganha aos trabalhadores nas negociações salariais, afetando a desigualdade de renda.

Estudos mais recentes, embasados em evidências empíricas, defendem que a relação entre desigualdade de renda e crescimento econômico assumiria, na verdade, um formato próximo a um "N" e não o "U-invertido da $\mathrm{CK}$, pois a desigualdade de renda voltaria a aumentar devido ao crescimento em níveis maiores de renda per capita. Os trabalhos que defendem a relação desigualdade-crescimento no formato " $\mathrm{N}$ " também o justificam pela abordagem da economia dual, destacando o papel da dinâmica da (des)industrialização. Segundo Bishop et al. (1991) e List e Gallet (1999), a volta do aumento da desigualdade de renda em níveis mais elevados de renda per capita decorreria da transição de uma economia com predominância industrial para uma economia baseada no setor de serviços - ocorrência de uma desindustrialização "natural”. Assim como na migração de trabalhadores da agropecuária para a indústria, a migração desta para os serviços elevaria desigualdade de renda devido ao diferencial de rendimento dos setores.

$\mathrm{Na}$ literatura, existem outras possíveis explicações para o "N". Katz e Murphy (1992), por exemplo, defendem que o aumento da desigualdade de renda com o crescimento em níveis maiores de renda decorre do aumento da demanda por trabalhadores mais qualificados devido a mudanças tecnológicas e expansões do comércio internacional, entre outros aspectos que alteram a dinâmica do mercado de trabalho. Já Glaeser (2005) e Piketty (2006) justificam o formato " $\mathrm{N}$ " por meio de questões tributárias, legislações trabalhistas e políticas redistributivas.

\section{Estratégias empíricas e dados}

Para avaliar como a industrialização afeta a distribuição dos rendimentos do trabalho nos municípios brasileiros, são feitas estimações em painel com dados municipais de 2000 e 2010 - últimos anos censitários. Os métodos empregados são: i) efeitos fixos - Mínimos Quadrados Ordinários (MQO) com erros-padrão robustos e estimador Within (variáveis em desvios em relação às suas médias para cada observação); e ii) efeitos aleatórios - Mínimos Quadrados Generalizados (MQG). O teste de Hausman é feito para verificar qual dos métodos é o adequado (BALTAGI, 2001; GREENE, 2011). Os modelos estimados baseiam-se na equação (1).

$$
\begin{aligned}
& D_{i t}=\alpha_{0}+\alpha_{1} V I_{i t}+\alpha_{2} V I_{i t}^{2}+\alpha_{3} E I_{i t}+\alpha_{4} E I_{i t}^{2}+\alpha_{5} Y_{i t}+\alpha_{6} Y_{i t}^{2}+\alpha_{7} Y_{i t}^{3}+\alpha_{8} X_{i t}+T_{t}+ \\
& u_{i}+\varepsilon_{i t}
\end{aligned}
$$

sendo: $D_{i t}$ a variável dependente do município $i$ no ano $t ; \alpha_{0}$ a constante; $V I_{i t}, E I_{i t}$ e $Y_{i t}$ as variáveis explicativas de interesse; $\alpha_{1}$ a $\alpha_{7}$ os coeficientes das variáveis de interesse; $X_{i t}$ o vetor de variáveis de controle; $\alpha_{8}$ o vetor de coeficientes dos controles; $T_{t}$ uma dummy para o ano de 2010 (2000 como default); $u_{i}$ os efeitos fixos; e $\varepsilon_{i t}$ o erro.

A variável dependente $D_{i t}$ é o índice de Theil-L dos rendimentos do trabalho para maiores de 18 anos (Theil-L trabalho). Este mede a desigualdade na distribuição de indivíduos ocupados de 18 anos de idade ou mais, pelo rendimento de todos os trabalhos, calculada pelo logaritmo da razão entre as médias aritmética e geométrica dos rendimentos individuais. É igual a zero quando não há desigualdade de renda e é tendente ao infinito quando a desigualdade tende ao máximo. Os índices para cada município brasileiro são calculados e disponibilizados por PNUD (2013), a partir de dados dos Censos Demográficos de 2000 e 2010 do Instituto Brasileiro de Geografia e Estatística (IBGE). A escolha deste índice decorre do estudo averiguar o efeito da participação relativa de um setor produtivo sobre a desigualdade. Assim, insere-se no debate sobre distribuição pessoal e setorial da renda, que a trata como função de atributos pessoais e setoriais que afetam a produtividade do trabalho - prêmios por atributos (ALVAREZ, 1996). 
Pelo conceito original, discutido por Rowthorn e Wells (1987) e Rowthorn e Ramaswamy (1997), desindustrialização é um processo de retração significativa e persistente da participação da indústria no emprego total da economia. Tregenna (2009) propõe um conceito ampliado para o processo: quedas concomitantes das participações industriais no emprego e produto. Segundo a autora, a definição de desindustrialização em termos apenas de emprego é limitada em função de vários fatores, destacando-se o fato de que um aumento da produtividade da indústria maior do que o observado nos serviços pode induzir "desindustrialização" somente pelo emprego.

Assim, optou-se por considerar tanto a participação do valor adicionado da indústria (VA indústria) no Produto Interno Bruto (PIB) do município $i$ no ano $t$ - razão entre o VA industrial e o PIB, representada em (1) por $V I_{i t}$-, como a participação da indústria no emprego (emprego indústria) - representada em (1) por $E I_{i t}$. Por se tratarem de participações relativas, suas interpretações são aumentos ou reduções do produto ou emprego industrial comparativamente ao agregado de outros setores econômicos - serviços, agropecuária e "público" (administração, saúde e educação públicas e seguridade social). As informações dos VAs setoriais e dos PIBs são oriundas do IBGE. Já a variável emprego indústria é calculada e disponibilizada por PNUD (2013), a partir de dados do IBGE, representando a razão entre as pessoas de 18 anos de idade ou mais ocupadas no setor industrial e o total de pessoas ocupadas da mesma faixa etária.

As participações da indústria no produto e no emprego são consideradas na equação (1) em funções quadráticas (polinômios de $2^{\circ}$ grau) $-V I_{i t}$ e $V I_{i t}^{2}$ e $E I_{i t}$ e $E I_{i t}^{2}$, respectivamente. Esta é a proposta deste estudo para testar a hipótese da abordagem da economia dual de que, em estágios iniciais da industrialização, a migração de trabalhadores da agropecuária para a indústria e o diferencial de aumento da produtividade do trabalho, maior na indústria, pioram a desigualdade de rendimentos do trabalho; em estágios mais avançados, a migração se estabiliza, a indústria se consolida e, assim, a desigualdade passa a diminuir em função da industrialização (KUZNETS, 1955; DEUTSCH; SILBER, 2000). Como já mencionado, tal hipótese é uma das justificativas da literatura para a hipótese da Curva de Kuznets (CK). Porém, aqui é proposta uma forma de testar especificamente o formato da relação desigualdade-industrialização.

Vale ressalvar que maiores participações da indústria no produto e emprego em relação aos serviços também implicam, pela abordagem da economia dual, em menor desigualdade de renda. Como discutido anteriormente, a expansão dos serviços em relação à indústria é uma das explicações da possível volta do aumento da desigualdade em níveis maiores de renda, o que justificaria a relação desigualdade-crescimento em "N" (BISHOP et al., 1991; LIST; GALLET, 1999). Outro aspecto a considerar é que evidências para o Brasil sinalizam diferenciais de rendimentos entre os setores público e privados, em favor do primeiro (MARCONI, 1997; BELLUZZO et al., 2005). Assim, é plausível esperar que quanto maiores as participações industriais no produto e emprego em relação ao setor público, menor a desigualdade de renda.

Para corroborar a hipótese da economia dual, as evidências devem sugerir uma relação não linear entre a desigualdade de rendimentos e a industrialização, em formato próximo a um "U-invertido". Ou seja, a desigualdade aumenta com a industrialização em níveis pequenos de participação industrial e diminui em níveis mais elevados. Para isso, os coeficientes estimados das variáveis em nível devem ser significativos e positivos; já os coeficientes das variáveis ao quadrado devem ser significativos e negativos. Assim, para as participações no produto e no emprego, respectivamente: i) $\alpha_{1}>0$ e $\alpha_{2}<0$; e ii) $\alpha_{3}>0$ e $\alpha_{4}<0$. A hipótese é corroborada, então, se as relações tiverem turning points (pontos de máximo). Derivando (1) em relação às variáveis, a participação industrial média no produto no turning point $(\overline{V I})$ é 
calculado pela equação (2); já a participação média no emprego no turning point $(\overline{E I})$, pela equação (3).

$$
\begin{aligned}
& \overline{V I}=-\alpha_{1} / 2 \alpha_{2} \\
& \overline{E I}=-\alpha_{3} / 2 \alpha_{4}
\end{aligned}
$$

Deve-se apontar que a relação entre industrialização e desigualdade de rendimentos pode advir de efeitos composição e concentração. Segundo Soares (2006), alterações na desigualdade de um local em dado período, medido por índices de concentração - como o Theil-L -, são sensíveis a tais efeitos. Para o escopo do presente estudo, o efeito composição é a variação da desigualdade provocada por alterações das participações dos setores produtivos. Já o efeito concentração é a variação da desigualdade por mudanças na concentração da renda nos setores. Por exemplo, se um setor com concentração de renda bastante distinta dos demais tem sua participação no emprego (ou produto) alterada, ocorre variação da desigualdade pelo efeito composição; já se um setor com grande participação apresenta variação de sua concentração de renda, ocorre alteração da desigualdade por efeito concentração (HOFFMANN; NEY, 2008).

Considerando evidências de outros trabalhos de que as atividades industriais tendem a concentrar menos os rendimentos do trabalho no Brasil do que os demais setores (MARCONI, 1997; BELLUZZO et al., 2005; HOFFMANN, 2011; OLIVEIRA; SILVEIRA NETO, 2016), seria plausível esperar que o aumento da participação industrial sempre resultaria em redução da desigualdade de renda nos municípios, pelo efeito composição. Contudo, se for constatada a relação não linear proposta, pode-se afirmar que a industrialização impacta a desigualdade de rendimentos do trabalho por combinações dinâmicas dos efeitos composição e concentração, o que estaria de acordo com as hipóteses da economia dual apresentadas na seção anterior.

A Tabela 1 mostra algumas estatísticas descritivas para as variáveis dependente $\left(D_{i t}\right)$ e explicativas de interesse $\left(V I_{i t}\right.$ e $\left.E I_{i t}\right)$ para os anos de 2000 e 2010. Observa-se que o índice de Theil-L dos rendimentos do trabalho municipal médio reduziu de 2000 a 2010, assim como seu desvio-padrão, sinalizando melhora da desigualdade no período. Já as participações industriais no produto (VA indústria) e emprego (emprego indústria), na média, aumentaram um pouco.

Como já mencionado, a hipótese da CK pressupõe uma relação entre a desigualdade de renda e o nível de renda per capita no formato de um "U-invertido"; ou seja, em níveis baixos de renda per capita, a desigualdade aumenta com o crescimento econômico; porém, atingido certo nível, a desigualdade passa a cair (KUZNETS, 1955; DEUTSCH; SILBER, 2000). Porém, evidências de alguns trabalhos contestam a CK, sinalizando que a queda da desigualdade com o crescimento econômico pode não se manter em níveis maiores de renda. Assim, a relação desigualdade-crescimento teria, na verdade, um formato próximo a um "N" (BISHOP et al., 1991; LIST; GALLET, 1999). Nesse sentido, uma contribuição secundária deste estudo é testar as hipóteses da $\mathrm{CK}$ e do formato "N", gerando evidências adicionais à literatura empírica.

Tabela 1 - Variáveis dependente e explicativas de interesse: estatísticas descritivas - 2000 e 2010

\begin{tabular}{c|c|c|c|c|c|c|c|c}
\hline \multirow{2}{*}{ Covariadas } & \multicolumn{4}{|c|}{$\mathbf{2 0 0 0}$} & \multicolumn{4}{c}{$\mathbf{2 0 1 0}$} \\
\cline { 2 - 9 } & Média & D.P. & Mínimo & Máximo & Média & D.P. & Mínimo & Máximo \\
\hline Theil-L Trabalho & 0,477 & 0,154 & 0,000 & 1,420 & 0,389 & 0,122 & 0,000 & 1,400 \\
VA indústria & 0,145 & 0,122 & 0,012 & 0,917 & 0,160 & 0,125 & 0,009 & 0,931 \\
Emprego Indústria & 0,158 & 0,099 & 0,003 & 0,739 & 0,167 & 0,096 & 0,009 & 0,701 \\
\hline
\end{tabular}

Fonte: PNUD (2013), IBGE. Elaboração própria. D.P.: desvio-padrão. 
Para isso, é inserido, como variável explicativa, o PIB per capita dos municípios ( $\mathrm{R} \$$ milhões de 2000) em uma função cúbica (polinômio de $3^{\circ}$ grau), como é ilustrado na equação (1) pelos termos $Y_{i t}, Y_{i t}^{2}$ e $Y_{i t}^{3}$. A hipótese do "N" é corroborada se os coeficientes associados ao PIB per capita forem significativos, positivos em nível e ao cubo $\left(\alpha_{5}>0\right.$ e $\left.\alpha_{7}>0\right)$ e negativo ao quadrado $\left(\alpha_{6}<0\right)$. Para confirmar o formato, deve ser realizado o teste da $2^{\mathrm{a}}$ derivada: i) obter a $1^{\text {a }}$ derivada de (1) em relação a $Y$, considerando os coeficientes estimados $\left(\alpha_{5}, \alpha_{6}\right.$ e $\left.\alpha_{7}\right)$; ii) calcular os pontos críticos médios; iii) obter a $2^{\mathrm{a}}$ derivada e substituir os pontos críticos nela. Se um dos valores for negativo e o outro positivo, há pontos de máximo e mínimo (CHIANG, 1982), corroborando o formato próximo a um "N". Já se $\alpha_{5}>0\left(Y_{i t}\right)$, $\alpha_{6}<0\left(Y_{i t}^{2}\right)$ e $\alpha_{7}<0\left(Y_{i t}^{3}\right)$ ou $\alpha_{7}$ não for significativo, a relação desigualdade-crescimento assume o formato da CK. Quaisquer outras combinações de sinais refutam os dois formatos. Os dados para o cálculo dos PIBs per capita são oriundos do IBGE. A Tabela 2 apresenta algumas estatísticas descritivas.

Tabela 2 - Covariadas: descrições e estatísticas descritivas - 2000 e 2010

\begin{tabular}{c|c|c|c|c|c}
\hline \multirow{2}{*}{ Covariadas } & \multirow{2}{*}{ Descriçães } & \multicolumn{2}{|c}{$\mathbf{2 0 0 0}$} & \multicolumn{2}{|c}{$\mathbf{2 0 1 0}$} \\
\cline { 3 - 6 } & Média & D.P. & Média & D.P. \\
\hline PIB per capita & PIB per capita $(\mathrm{R} \$$ milhões de 2000) & 0,004 & 0,005 & 0,002 & 0,003 \\
\hline Instrução & \% ocupados com ensino médio - 18 anos ou mais & 0,169 & 0,087 & 0,304 & 0,098 \\
\hline População & População - milhares habitantes & 30,76 & 186,62 & 33,83 & 200,28 \\
\hline Urbanização & \% população urbana & 0,589 & 0,233 & 0,638 & 0,220 \\
\hline Mulheres & \% de mulheres na população & 0,492 & 0,014 & 0,495 & 0,016 \\
\hline Não Brancos & \% de não brancos na população & 0,475 & 0,255 & 0,533 & 0,242 \\
\hline Jovens & \% população de 18 anos ou mais & 0,618 & 0,060 & 0,685 & 0,056 \\
\hline Formalização & \% ocupados formais - 18 anos ou mais & 0,361 & 0,181 & 0,435 & 0,193 \\
\hline
\end{tabular}

Fonte: PNUD (2013), IBGE. Elaboração própria. D.P.: desvio-padrão.

O controle pelo PIB per capita pode suavizar um potencial viés de omissão de variáveis explicativas importantes, pois este é correlacionado a várias dimensões do desenvolvimento econômico que afetam a desigualdade de renda. Ademais, ao ser controlado em uma função, é possível, pode captar os efeitos dos outros fatores, discutidos na seção anterior, que explicariam a relação entre desigualdade de renda e crescimento econômico, tanto no formato da CK como em "N", de modo que as prováveis relações encontradas entre a desigualdade de rendimentos do trabalho e as participações industriais no produto e no emprego possam ser interpretadas, com maior robustez, como efeitos causais da industrialização sobre a desigualdade de renda.

Uma recomendação da literatura sobre a CK, adotada neste estudo, é o controle por outras características observadas dos locais - municípios - que também podem afetar a distribuição da renda e que são variantes no tempo (AHLUWALIA, 1976; ANAND; KANBUR, 1993; JHA, 1996). A Tabela 2 apresenta o vetor de variáveis de controle (covariadas) $X_{i t}$ considerado nas estimações, suas descrições e estatísticas descritivas para 2000 e 2010. Todas as variáveis são oriundas do PNUD (2013), que as calculou por meio de informações censitárias do IBGE.

Em relação às variáveis instrução e jovens, deve-se apontar que a literatura fundamentada na Teoria do Capital Humano e na equação Minceriana de salários defende que o aumento da média de pessoas com maiores níveis de educação e idade (proxy para experiência) melhoram a distribuição da renda. Porém, existiriam pontos de inflexões que geram saltos no rendimento, como por exemplo, a conclusão de ciclos de educação e o alcance da maior idade (MINCER, 1974; ALVAREZ, 1996). Assim, optou-se pelo ensino médio como referência por ser um dos mais importantes pontos de inflexão quando se trata de 
educação e a faixa etária acima de 18 anos por ser a idade em que as pessoas normalmente entram no mercado de trabalho formal.

No Brasil, a literatura sinaliza que trabalhadores de áreas urbanas tendem a ser melhor remunerados e que há diferenciais de salários entre mulheres e homens e entre não brancos e brancos, o que decorre de discriminações por gênero e etnia/cor (BARROS et al., 2007). Assim, é importante controlar as variáveis urbanização, mulheres e não brancos para captarem os efeitos das distribuições dos trabalhadores (espacial, por gênero e etnia/cor) na desigualdade.

Em relação à variável formalização, que representa os trabalhadores que possuem carteira de trabalho assinada, Maia (2013) mostra que estes possuem, no Brasil, remuneração média maior do que os trabalhadores ocupados em posições informais. Ademais, outra constatação é que a formalização melhora a distribuição de renda. A variável população, em conjunto com as demais, controla o tamanho do mercado de trabalho em cada um dos municípios brasileiros.

Além das covariadas, também são controladas: i) características não observadas distintas entre os municípios e constantes no tempo - efeitos fixos $\left(\mu_{i}\right)-$; e ii) características constantes entre os municípios e variantes no tempo - efeito fixo temporal $\left(T_{t}\right)$, controlado pela dummy para o ano de 2010. De acordo com a literatura, atributos históricos, institucionais e culturais específicos de cada local influenciam a desigualdade de renda (DEUTSCH; SILBER, 2000).

Para averiguar a robustez dos resultados frente a possíveis correlações entre as variáveis explicativas de interesse (multicolinearidade), são estimados modelos para 3 especificações. A especificação I representa o modelo completo, como expresso na equação (1). Na especificação II, é retirado o polinômio de $2^{\circ}$ grau referente à participação industrial no emprego, mantendo a função quadrática da participação industrial no produto, a função cúbica do PIB per capita, as covariadas e a dummy 2010. Na especificação III, são controlados: a função quadrática da participação da indústria no emprego, a função cúbica do PIB per capita, as demais covariadas e a dummy 2010; ou seja, não é considerada na estimação a participação industrial no produto.

Com o intuito de avaliar se as possíveis relações entre a desigualdade dos rendimentos do trabalho e as participações industriais no produto e no emprego não são lineares, mas com um formato distinto do proposto, é estimado um modelo baseado na equação (2) especificação $I V$-, nas quais as participações industriais são consideradas em funções cúbicas - $V I_{i t}, V I_{i t}^{2}$ e $V I_{i t}^{3}$ e $E I_{i t}, E I_{i t}^{2}$ e $E I_{i t}^{3}$. A combinação dos coeficientes em nível, ao quadrado e ao cubo e, se for o caso, testes da $2^{\mathrm{a}}$ derivada apontarão os formatos obtidos para as relações. Se os coeficientes das participações em nível e ao quadrado respeitarem o padrão para inferir o "U-invertido" e os coeficientes ao cubo $\left(\alpha_{9}\right.$ e $\left.\alpha_{10}\right)$ forem negativos ou não significativos, tal formato é confirmado.

$D_{i t}=\alpha_{0}+\alpha_{1} V I_{i t}+\alpha_{2} V I_{i t}^{2}+\alpha_{9} V I_{i t}^{3}+\alpha_{3} E I_{i t}+\alpha_{4} E I_{i t}^{2}+\alpha_{10} E I_{i t}^{3}+\alpha_{5} Y_{i t}+\alpha_{6} Y_{i t}^{2}+$ $\alpha_{7} Y_{i t}^{3}+\alpha_{8} X_{i t}+T_{t}+u_{i}+\varepsilon_{i t}$

Uma crítica que as estimações até aqui propostas podem sofrer se refere ao índice TheilL do trabalho variar de 0 ao infinito. Assim, sofre censura no limite inferior. Na presença de censura na variável dependente, as estimações por MQO, como no painel com efeitos fixos, podem ser viesadas e inconsistentes. Uma opção é o método Tobit em painel, com estimação por máxima verossimilhança. Tal método é vantajoso por usar distribuição mais adequada para dados censurados. Porém, não controla efeitos fixos e os coeficientes não são efeitos médios ou marginais, mas os sinais podem ser interpretados (BALTAGI, 2001; GREENE, 2011).

Assim, para averiguar a robustez dos resultados frente à censura, são estimados modelos baseados em (1) e (2) - especificações $I$ e $I V$-, desconsiderando os efeitos fixos $\left(\mu_{i}\right)$, por 
Tobit em painel. Devido às especificidades do método, não são mensurados os turning points, mas é possível inferir os formatos das relações entre desigualdade de renda, participações industriais e PIB pelos sinais e significâncias dos coeficientes, de acordo com os padrões já apontados.

Os métodos já propostos são para estimações paramétricas. Para evidências adicionais, é interessante usar regressões não paramétricas para "ajustar" curvas que representam as relações entre a desigualdade dos rendimentos e as participações industriais. O método escolhido é o de Kernel-Weighted Local Polynomial Regression, que permite a visualização gráfica das relações entre variáveis sem a imposição prévia de uma forma funcional, com os "dados falando por si mesmos". Nestas regressões, para ajustar melhor as relações, as variáveis Theil-L trabalho, VA indústria e emprego indústria são consideradas em logaritmo natural $(\ln )$ e apenas para 2010.

\section{Análises dos resultados}

A seguir, são analisados os resultados que possibilitam averiguar como a industrialização afeta a distribuição dos rendimentos do trabalho nos municípios brasileiros. Para não fugir do escopo do estudo, os coeficientes das covariadas e da dummy 2010 não são reportados, assim os coeficientes estimados por efeitos aleatórios, pois os testes de Hausman sinalizam, em todas as estimações, a melhor adequação dos efeitos fixos (estatísticas dos testes são significativas) $)^{9}$.

A Tabela 3 mostra os resultados das estimações paramétricas. Os coeficientes estimados associados à função quadrática da participação da indústria no produto (VA indústria), em todas as estimações em que é considerada, são significativos, sendo positivos em nível e negativos ao quadrado. Já os coeficientes para a variável ao cubo não são significativos (especificação IV). Tais resultados são constatados tanto por efeitos fixos como por Tobit em painel, sugerindo que a relação desigualdade-industrialização, pelo lado do produto, tem um formato próximo a um "U-invertido". Ou seja, que a desigualdade dos rendimentos do trabalho aumenta em função da industrialização até certa participação industrial no produto (turning point), a partir da qual passa a cair. Na especificação I, o turning point $(\overline{V I})$ é em uma participação média da indústria no produto de $23,1 \%$; na $I I$, o turning point é em $22,5 \%$; e na $I V$, o turning point é em $18,6 \%$.

Pelo lado do emprego, os resultados também são favoráveis à relação entre desigualdade de rendimentos do trabalho e industrialização em "U-invertido". Os coeficientes associados à função quadrática da variável emprego indústria, em todas as estimações em que é considerada, são significativos, positivos em nível e negativos ao quadrado. Ademais, os coeficientes para a variável ao cubo não são significativos (especificação $I V$ ). Estes resultados são observados por efeitos fixos e por Tobit em painel. Assim, a desigualdade dos rendimentos do trabalho aumenta devido à industrialização até dada participação industrial no emprego (turning point), a partir da qual cai. Na especificação I, o turning point $(\overline{E I})$ é em uma participação industrial média no emprego de $18,3 \%$; na $I I I$, o turning point é em 18,6\%; e na $I V$, o turning point é em $12,6 \%$.

As participações médias da indústria no produto e no emprego em 2010 nos municípios brasileiros era de $16 \%$ e 16,7\%, respectivamente (Tabela 1). Estas participações são inferiores aos respectivos turning points em todas as estimações. Assim, pode-se inferir que processos de industrialização nos municípios, com aumento da participação no produto e no emprego, ceteris paribus, tenderão a aumentar, inicialmente, a desigualdade dos rendimentos do

\footnotetext{
${ }^{8}$ Para mais detalhes, ver, entre outros: Cleveland e Devlin (1988), Jones et al. (1994) e Loader (1999).

${ }^{9}$ Os resultados não reportados podem ser solicitados aos autores.
} 
trabalho; mas, à medida que tais processos se aprofundarem, os turning points serão atingidos e a desigualdade passará a cair. Comparando os turning points calculados do VA $(\overline{V I})$ e do emprego $(\overline{E I})$, pode-se afirmar que a redução da desigualdade ocorre antes com o avanço do emprego industrial.

Tabela 3 - Resultados: estimações por painel com efeitos fixos e Tobit em painel

\begin{tabular}{|c|c|c|c|c|c|c|c|}
\hline \multirow{3}{*}{\multicolumn{2}{|c|}{$\begin{array}{l}\text { Variáveis / } \\
\text { Estatísticas }\end{array}$}} & \multicolumn{6}{|c|}{ Métodos / Especificações } \\
\hline & & \multicolumn{4}{|c|}{ Efeitos Fixos } & \multicolumn{2}{|c|}{ Tobit } \\
\hline & & $\mathbf{I}$ & II & III & IV & I & IV \\
\hline \multicolumn{2}{|c|}{ VA Indústria } & $\begin{array}{l}0,281^{(a)} \\
(0,074)\end{array}$ & $\begin{array}{l}0,274^{(a)} \\
(0,074)\end{array}$ & & $\begin{array}{l}0,443^{(a)} \\
(0,142)\end{array}$ & $\begin{array}{l}0,054^{(\mathrm{c})} \\
(0,022)\end{array}$ & $\begin{array}{l}0,308^{(a)} \\
(0,078)\end{array}$ \\
\hline \multicolumn{2}{|c|}{ VA Indústria² } & $\begin{array}{l}-0,608^{(a)} \\
(0,116)\end{array}$ & $\begin{array}{l}-0,608^{(a)} \\
(0,115)\end{array}$ & & $\begin{array}{l}-1,191^{\text {(a) }} \\
(0,441)\end{array}$ & $\begin{array}{c}-0,246^{(a)} \\
(0,057)\end{array}$ & $\begin{array}{c}-1,101^{(a)} \\
(0,250)\end{array}$ \\
\hline \multicolumn{2}{|c|}{ VA Indústria ${ }^{3}$} & & & & $\begin{array}{c}0,518 \\
(0,393)\end{array}$ & & $\begin{array}{c}0,744 \\
(0,719)\end{array}$ \\
\hline \multicolumn{2}{|c|}{ Emprego Indústria } & $\begin{array}{l}0,249^{(\mathrm{a})} \\
(0,095)\end{array}$ & & $\begin{array}{l}0,263^{(\mathrm{a})} \\
(0,095)\end{array}$ & $\begin{array}{l}0,622^{(a)} \\
(0,182)\end{array}$ & $\begin{array}{l}0,173^{(\mathrm{a})} \\
(0,049)\end{array}$ & $\begin{array}{l}0,836^{(\mathrm{a})} \\
(0,098)\end{array}$ \\
\hline \multicolumn{2}{|c|}{ Emprego Indústria ${ }^{2}$} & $\begin{array}{l}-0,680^{(a)} \\
(0,188)\end{array}$ & & $\begin{array}{l}-0,708^{(a)} \\
(0,188)\end{array}$ & $\begin{array}{l}-2,463^{(a)} \\
(0,717)\end{array}$ & $\begin{array}{l}-0,649^{(a)} \\
(0,091)\end{array}$ & $\begin{array}{c}-3,698^{(a)} \\
(0,391)\end{array}$ \\
\hline \multicolumn{2}{|c|}{ Emprego Indústria ${ }^{3}$} & & & & $\begin{array}{c}2,293 \\
(2,814)\end{array}$ & & $\begin{array}{c}3,735 \\
(3,454)\end{array}$ \\
\hline \multicolumn{2}{|c|}{ PIB per capita } & $\begin{array}{c}27,164^{(a)} \\
(2,327)\end{array}$ & $\begin{array}{c}26,840^{(a)} \\
(2,319)\end{array}$ & $\begin{array}{c}23,389^{(a)} \\
(2,362)\end{array}$ & $\begin{array}{c}26,942^{(a)} \\
-2,353\end{array}$ & $\begin{array}{c}19,421^{\text {(a) }} \\
(1,147)\end{array}$ & $\begin{array}{c}20,559^{(a)} \\
(1,1780\end{array}$ \\
\hline \multicolumn{2}{|c|}{ PIB per capita ${ }^{2}$} & $\begin{array}{c}-525,070^{(a)} \\
(75,333)\end{array}$ & $\begin{array}{c}-519,651^{(a)} \\
(74,620)\end{array}$ & $\begin{array}{c}-478,104^{(a)} \\
(75,271)\end{array}$ & $\begin{array}{c}-528,551^{(a)} \\
(78,083)\end{array}$ & $\begin{array}{c}-460,190^{(a)} \\
(39,773)\end{array}$ & $\begin{array}{c}-497,058^{(a)} \\
(41,068)\end{array}$ \\
\hline \multicolumn{2}{|c|}{ PIB per capita ${ }^{3}$} & $\begin{array}{c}2.792,592^{(a)} \\
(529,629)\end{array}$ & $\begin{array}{c}2.766,093^{(a)} \\
(525,122)\end{array}$ & $\begin{array}{c}2.625,225^{(a)} \\
(525,237)\end{array}$ & $\begin{array}{c}2.828,810^{(a)} \\
(550,595)\end{array}$ & $\begin{array}{c}2.623,265^{(a)} \\
(283,556)\end{array}$ & $\begin{array}{c}2.858,801^{(\mathrm{a})} \\
(291,813)\end{array}$ \\
\hline \multicolumn{2}{|c|}{ Dummy 2010} & Sim & Sim & Sim & Sim & Sim & Sim \\
\hline \multicolumn{2}{|c|}{ Covariadas } & Sim & Sim & Sim & Sim & Sim & Sim \\
\hline \multicolumn{2}{|c|}{ Prob $>F$} & 0,000 & 0,000 & 0,000 & 0,000 & --- & --- \\
\hline \multicolumn{2}{|c|}{ Prob > chi ${ }^{2}$} & --- & --- & --- & --- & 0,000 & 0,000 \\
\hline \multicolumn{2}{|c|}{ Teste de Hausman } & $193,94^{(\mathrm{a})}$ & $200,75^{(a)}$ & $196,16^{(\mathrm{a})}$ & $193,94^{(a)}$ & --- & --- \\
\hline \multicolumn{2}{|c|}{ Observações } & 11.069 & 11.069 & 11.069 & 11.069 & 11.069 & 11.069 \\
\hline \multirow{2}{*}{$\begin{array}{l}\text { Turning } \\
\text { Points }\end{array}$} & $\overline{V I}$ & 0,231 & 0,225 & --- & 0,186 & --- & -- \\
\hline & $\overline{E I}$ & 0,183 & --- & 0,186 & 0,126 & --- & --- \\
\hline
\end{tabular}

Fonte: PNUD (2013), IBGE. Elaboração própria.

Erros-padrão robustos entre parênteses. ${ }^{(a)}$ Significativo a $1 \%$. (b) Significativo a 5\%. ${ }^{\text {(c) }}$ Significativo a $10 \%$.

Em relação aos coeficientes estimados associados à função cúbica do PIB per capita, a hipótese da CK (relação desigualdade-renda em "U-invertido") não é corroborada em todas as estimações. Os coeficientes associados ao PIB per capita são significativos, positivos em nível e negativos ao quadrado, como preconiza a hipótese. Porém, os coeficientes associados ao PIB per capita ao cubo são significativos e positivos, o que sinaliza que a relação desigualdade-crescimento tem formato próximo a um " $N$ ". Os testes da $2^{\text {a }}$ derivada confirmam tal formato ${ }^{10}$.

Por último, a Figura 1 apresenta as relações "ajustadas" pelo método de regressão não paramétrica Kernel-Weighted Local Polynomial Regression. O Gráfico (a) mostra a relação entre a desigualdade de renda (Theil-L trabalho) - eixo vertical - e a participação industrial no emprego (emprego indústria) - eixo horizontal -. Observa-se que a desigualdade aumenta até certo nível de participação industrial, a partir do qual diminui, o que se configura como outra evidência para corroborar a relação em "U-invertido" sugerida pelas estimações paramétricas.

\footnotetext{
${ }^{10}$ Os testes e os pontos críticos não são reportados, mas podem ser obtidos junto aos autores.
} 
Já na participação industrial no produto (VA indústria), o Gráfico (b) sinaliza que, com oscilações, a desigualdade dos rendimentos do trabalho (Theil-L trabalho) - eixo vertical aumenta até certo estágio de participação industrial - eixo horizontal -, a partir do qual passa a diminuir. Pode-se questionar que existe uma aparente reversão da tendência de queda em níveis mais avançados de participação industrial no produto; porém, vale ressalvar que a desigualdade de renda não atinge patamar similar ao observado em níveis intermediários de participação, o que não permite refutar integralmente a hipótese da relação desigualdadeindustrialização em "U-invertido", ainda mais por esta ter sido sinalizada em todas as estimações paramétricas.

\section{Figura 1 - Relações "ajustadas" entre a desigualdade de rendimentos e as participações industriais no emprego (a) e no produto (b) - 2010}

(a) Participação no Emprego

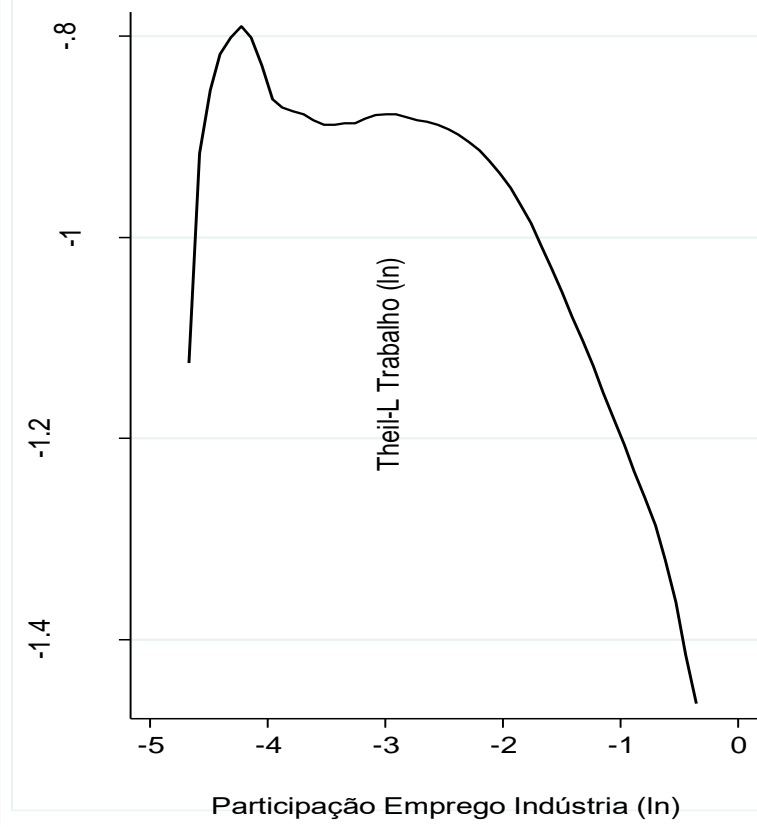

(b) Participação no Produto

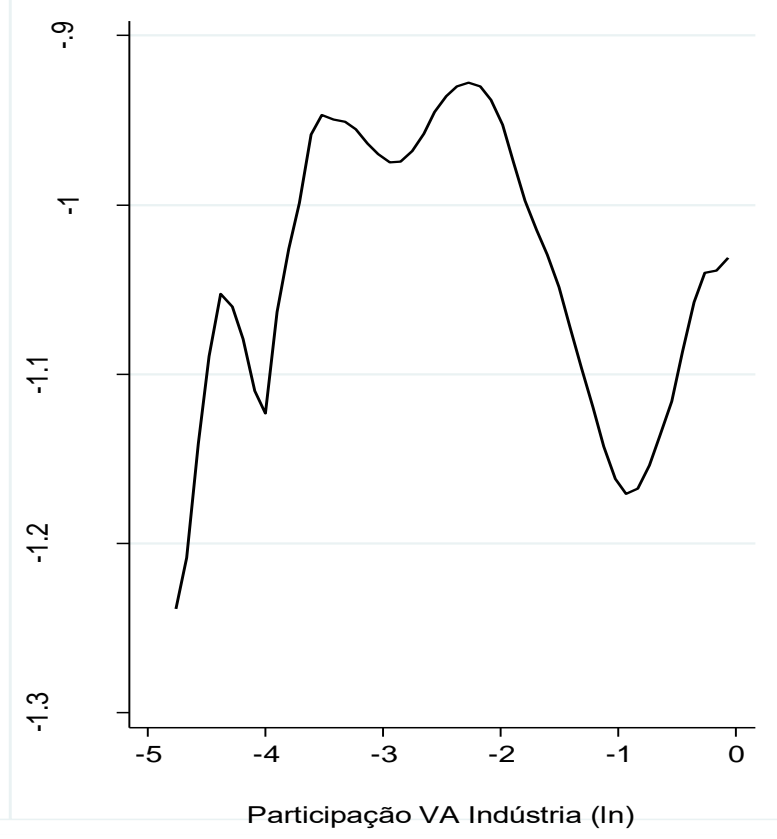

Fonte: PNUD (2013), IBGE. Elaboração própria.

\section{Considerações finais}

A distribuição da renda e a industrialização são temas recorrentes e de suma importância ao desenvolvimento econômico. Discussões da academia brasileira e de âmbito mundial, que são sistematizados no início deste estudo, tentam explicar as interfaces destas duas dimensões. Nesse sentido, uma das vertentes que emergem é da economia dual, que defende a dinâmica da composição setorial da produção de uma economia como condicionante da desigualdade. Em uma primeira fase, a transição de uma economia baseada em atividades de agropecuária para uma predominantemente industrial elevaria a desigualdade de renda. Porém, uma vez consolidada a indústria, a desigualdade cairia. Em uma segunda fase, a transição da economia industrial para uma baseada em serviços geraria novo aumento da desigualdade. Assim, a industrialização, tanto frente à agropecuária como ao setor de serviços, melhoraria a concentração de renda.

Tal hipótese foi testada e corroborada neste estudo por meio de estimações econométricas paramétricas em painel (efeitos fixos e efeitos aleatórios) e não paramétricas (método Kernel-Weighted Local Polynomial Regression) para todos os municípios brasileiros com informações disponibilizadas referentes aos anos de 2000 e 2010. Utilizando como 
medida de desigualdade o Theil-L dos rendimentos do trabalho, constatou-se que a desigualdade aumenta em função da industrialização até certas participações da indústria, a partir das quais, passa a cair. Portanto, as evidências sinalizam, com certa robustez, que a relação desigualdade-industrialização tem, nos municípios brasileiros, um formato próximo a um "U-invertido", tanto para a participação industrial no produto como no emprego. Adicionalmente, observou-se que a relação entre a desigualdade dos rendimentos e o crescimento econômico nos municípios brasileiros apresenta um formato próximo a um "N", contestando o tradicional formato da CK ("U-invertido").

A contestação do formato de "U-invertido" ocorre ao se observar que, em níveis maiores de PIB per capita, acontece uma aparente reversão da tendência de queda da desigualdade de renda, que passa a se elevar. Nesse momento, é possível identificar a formação de uma figura que se assemelha a um " $N$ ". Esse aumento da desigualdade de renda em consequência do aumento do PIB per capita decorre da expansão do setor de serviços, diversificação das atividades econômicas dos municípios, com surgimento, simultaneamente, de atividades intensivas em tecnologia e intensivas em mão de obra, o que provoca o alargamento da distribuição, como pela concentração de população pobre em municípios maiores.

Estes resultados, além de contribuírem para o preenchimento de uma lacuna na literatura empírica para dados de munícipios brasileiros, garantem evidências adicionais à literatura que tenta explicar a complexa cadeia causal entre processo de industrialização, desigualdade de renda, crescimento e desenvolvimento econômicos. Vale ressalvar que o estudo se preocupou em garantir evidências ainda inéditas que podem motivar trabalhos futuros mais aprofundados, que considerem além da participação setorial, por exemplo, a especialização e a produtividade em cada setor e suas interfaces com outros setores, como é o caso das agroindustriais.

\section{Referências bibliográficas}

AGHION, P.; BOLTON, P. "Distribution and growth in models of imperfect capital markets". European Economic Review, n.36, 1992.

AHLUWALIA, M. S. "Income distribution and development: some stylized facts". The American Economic Review, v.66, n.2, 1976.

ALVAREZ, A. R. Desenvolvimentos teóricos sobre distribuição de renda com ênfase em seus limites. Tese de Doutorado. USP, 1996.

ANAND, S.; KANBUR, S. M. R. "The Kuznets process and the inequality-development relationship". Journal of Development Economics, v.40, 1993.

BACHA, E. L. "Hierarquia e remuneração gerencial". In: TOLIPAN, R.; TINELLI, A. C. (coords.). A controvérsia sobre a distribuição de renda e desenvolvimento. Zahar, 1978.

BACHA, E. L.; TAYLOR, L. "Brazilian income distribution in the 1960s: acts, model results, and the controversy". In: TAYLOR, L.; BACHA, E. L.; CARDOSO, E. A.; LYSY, F. J. (eds.). Models of growth and distribution for Brazil. Oxford University Press, 1980.

BAER, M. O rumo perdido: a crise fiscal e financeira do Estado brasileiro. Paz e Terra, 1993.

BALTAGI, B. H. Econometric analysis of panel data. Wiley and Sons, 2001.

BARROS, R. P.; FOGUEL, M. N.; ULYSSEA, G. (orgs). Desigualdade de renda no Brasil: uma análise da queda recente (volumes 1 e 2). IPEA, 2007. 
BARROS, R. P.; HENRIQUES, R.; MENDONCA, R. "Pelo fim das décadas perdidas: educação e desenvolvimento sustentado no Brasil". In: HENRIQUES, R. (org.). Desigualdade e pobreza no Brasil. IPEA, 2000.

BELluZZO, W.; ANUATTI NETO, F.; PAZELlO, E. T. "Distribuição de salários e o diferencial público-privado no Brasil”. Revista Brasileira de Economia, v.59, n.4, 2005.

BÊRNI, D. A.; MARQUETTI, A.; KLOECKNER, R. "A desigualdade econômica no Rio Grande do Sul: primeiras investigações sobre a curva de Kuznets". Ensaios FEE, v.23, 2002.

BISHOP, J. A.; FORMBY, J. P.; THISTLE, P. D. "Changes in the US earnings distributions in the 1980s". Applied Economics, v.23, n.3, 1991.

BONELLI. R.; RAMOS. L. "Distribuição de renda no Brasil: avaliação das tendências de longo prazo e mudanças na desigualdade desde meados dos anos 70". Revista de Economia Política, v.13, n.2, 1993.

CANO, W. Desequilíbrios regionais e concentração industrial no Brasil - 1930/1970. Tese de Livre-Docência, Universidade Estadual de Campinas, 1981.

CANO, W. "Concentração e desconcentração econômica regional no Brasil". Economia e Sociedade, n.8, 1997.

CARDOSO, J. C.; POCHMANN, M. Raízes da concentração de renda no Brasil: 1930 a 2000. IPEA/CESIT, 2000.

CARNEIRO, D. D. “Crise e esperança: 1974-1980”. In: ABREU, M. P. (org). A Ordem do Progresso, Campus, $15^{\mathrm{a}} \mathrm{ed} ., 1990$.

CHIANG, A. Matemática para economistas. Makron Books, 1982.

CLEVELAND, W.; DEVLIN, S. "Locally weighted regression: an approach to regression analysis by local fitting". Journal of the American Statistical Association, v.83, 1988.

DEDECCA. C. S. A redução da desigualdade e seus desafios. IPEA. 2015.

DEUTSCH, J., SILBER, J. "The Kuznets curve and the impact of various income sources on the link between inequality and development". Working Paper Bar-Ilan University, 2000.

FIGUEIREDO, E. A.; SILVA JUNIOR, J. C. A.; JACINTO, P. A. “A hipótese de Kuznets para os municípios brasileiros: testes para as formas funcionais e estimações nãoparamétricas". Revista Economia, v.12, n.1, 2011.

FISHLOW, A. "Brazilian size distribution of income". American Economic Review, 1972.

FISHLOW, A. "A distribuição de renda no Brasil". In: TOLIPAN, R.; TINELLI, A. C. (coords.). A controvérsia sobre a distribuição de renda e desenvolvimento. Zahar, 1978.

FONSECA, P. C. D. "Sobre a intencionalidade da política industrializante do Brasil na década de 1930". Revista de Economia Política, v.23, 2003.

FURTADO, C. Formação econômica do Brasil. Editora Nacional, 7 aed., 1967.

GALOR, O.; TSIDDON, D. "Income distribution and growth: the Kuznets hypothesis revisited". Economica, v.63, n.250, 1996.

GANDRA, R. "O debate sobre a desigualdade de renda no Brasil: da controvérsia dos anos 70 ao pensamento hegemônico nos anos 90". História Econômica \& História de Empresas, v.III, n.1, 2005.

GLAESER, E. L. “Inequality”. NBER Working Paper Series, 2005. 
GREENE, W. H. Econometric analysis, Prentice Hall, 7ªed., 2011.

HOFFMANN, R. "Distribuição de renda e crescimento econômico". Estudos Avançados, n. 15, v.41, 2001.

HOFFMANN, R. "Distribuição da renda agrícola e sua contribuição para a desigualdade de renda no Brasil". Revista de Política Agrícola, v.20, n.2, 2011.

HOFFMANN, R.; DUARTE, J. C. “A distribuição da renda no Brasil”. Revista de Administração de Empresas, v.12, n.2, 1972.

HOFFMANN, R.; NEY, M. G. “A recente queda da desigualdade de renda no Brasil: análise de dados da PNAD, do Censo Demográfico e das Contas Nacionais”. Econômica, v.10, n.1, 2008 .

JHA, S. K. “The Kuznets Curve: a reassessment”. World Development, v.24, n.4, 1996.

JONES, M. C.; DAVIES, S. J.; PARK, B. U. "Versions of Kernel-type regression estimators". Journal of the American Statistical Association, v.89, n.427, 1994.

KALDOR, N. Causes of the slow rate of economic growth of the United Kingdom: an inaugural lecture. Cambridge University Press, 1966.

KATZ, L.; MURPHY, K. "Changes in relative wages, 1963-1987: supply and demand factors". Quarterly Journal of Economics, 1992.

KUZNETS, S. "Economic growth and income inequality". The American Economic Review, v.45, n.1, 1955.

LAGO, L. A. C. “A retomada do crescimento e as distorções do 'Milagre': 1967 - 1973”. In: ABREU, M. P. (org). A Ordem do Progresso, Campus, 15ªd., 1990.

LANGONI, C. G. "Distribuição da renda e desenvolvimento econômico do Brasil: uma reafirmação". Ensaios Econômicos EPGE, n.7, 1973.

LEWIS, W. A. "Economic development with unlimited supplies of labor". Manchester School of Economics and Social Studies, v.22, 1954.

LIST, J. A.; GALLET, C. A. "The Kuznets curve: what happens after the inverted-U”. Review of Development Economics, v.3, n.2, 1999.

LOADER, C. Local regression and likelihood. Springer, 1999.

MAIA. A. G. "Estrutura de ocupações e distribuição de rendimentos: uma análise da experiência brasileira nos anos 2000”. Revista de Economia Contemporânea. v.17. n.2, 2013.

MARCONI, N. "Uma breve comparação entre os mercados de trabalho do setor público e privado". Revista do Serviço Público, v.48, n.1, 1997.

MELLO, J. M. C. O capitalismo tardio: contribuição à revisão crítica da formação e do desenvolvimento da economia brasileira. Brasiliense, São Paulo, 1986.

MINCER, J. Schooling, experience, and earnings. Columbia University Press, 1974.

OLIVEIRA, R.; SILVEIRA NETO, R. M. "Estrutura produtiva ou escolaridade? Uma análise dos fatores explicativos da desigualdade de renda entre as regiões Sudeste e Nordeste por quantil para o período entre os anos de 1970 e 2010". Revista Econômica do Nordeste, v.47, n.3, 2016.

OREIRO, J. L.; FEIJÓ, C. A. "Desindustrialização: conceituação, causas, efeitos e o caso brasileiro". Revista de Economia Política, v.30, n.2, 2010. 
PIKETTY, T. "The Kuznets Curve: yesterday and tomorrow". In: BANERJEE, A.; BENABOU, R.; MOOKHERJEE, D. (eds.). Understanding poverty. Oxford Press, 2006.

PNUD. Atlas do desenvolvimento humano no Brasil. PNUD, 2013.

PREBISCH, R. "O desenvolvimento econômico da América Latina e alguns de seus problemas principais". Boletín económico de América Latina, CEPAL, v. VII, n. 1, 1962.

RAMOS, L. "Desigualdade de renda e crescimento econômico no Brasil: 1976/85". Textos para Discussão do IPEA, n.213, 1991.

ROCHA, L. E. V.; SANTOS, G. C.; ROSADO, P. L. "Indicadores de desigualdade de renda e pobreza na agricultura do Estado de Minas Gerais". Organizações Rurais \& Agroindustriais, v.15, n.3, 2013.

ROWTHORN, R.; RAMASWAMY, R. Deindustrialization: causes and implications. Working Paper, International Monetary Fund, n.97, v.42, 1997.

ROWTHORN, R; WELLS, J. R. De-industrialization and foreign trade. Cambridge University Press, 1987.

SABOIA, J. "Descentralização industrial no Brasil na década de noventa: um processo dinâmico e diferenciado regionalmente". Nova Economia, v.11, n.2, 2001.

SILVA, H. C. M. "Deterioração dos termos de intercâmbio, substituição de importações, industrialização e substituição de exportações: a política de comércio exterior brasileira de 1945 a 1979”. Revista Brasileira de Política Internacional, v.46, n.1, 2003.

SOARES, S. S. D. "Distribuição de renda no Brasil de 1976 a 2004 com ênfase no período entre 2001 e 2004". Textos para Discussão do IPEA, n.1166, 2006.

TAVARES, M. C. Da substituição de importações ao capitalismo financeiro: ensaios sobre economia brasileira. Zahar, 1975.

TREGENNA, F. "Characterizing deindustrialization: an analysis of changes in manufacturing employment and output internationally". Cambridge Journal of Economics, v.33, 2009. 